\title{
Getting quantifying-into questions uniformly: Functionality, domain exhaustivity, and quantificational variability *
}

\author{
Yimei Xiang \\ Rutgers University
}

\begin{abstract}
Questions with a quantifier have readings that seem to involve quantificationinto questions (QIQ). This paper provides a uniform compositional analysis of these readings. I propose that QIQ-readings are special functional readings - the question nucleus involves a functional dependency between the quantifier and $w h$-trace. I argue that the seeming QIQ-effect is derived by extracting a minimal set that satisfies a quantificational predication condition. The possible values of this minimal set determine whether QIQ-readings are available and whether a question admits a pair-list answer and/or a choice answer. This analysis also explains the contrast between $\forall$-questions and multi-wh questions with respect to domain exhaustivity, and accounts for the quantificational variability effects in question-embeddings.
\end{abstract}

Keywords: questions, quantification, pair-list, functionality, uniqueness, domain exhaustivity, quantificational variability, categorial approach, compositionality

\section{Introduction}

Questions with a universal quantifier (called $\forall$-questions) can be ambiguous between individual readings, functional readings, and pair-list readings (Engdahl 1980). As exemplified in (1), the three readings expect answers naming an individual book, a Skolem function to atomic books, and a list of kid-book pairs, respectively.

(1) Which book did every/each kid read?

a. Individual: 'For which book $y$ is s.t. every kid read $y$ ?'

b. Functional: 'For which function $\mathbf{f}$ to book is s.t. every kid $x \operatorname{read} \mathbf{f}(x)$ ?'

c. Pair-list: 'For every kid $x$, [tell me] which book did $x$ read?'

There are two general ways to think about the nature of the pair-list reading (1c). One way regards this reading as involving quantification-into questions (QIQ

* For helpful discussions, I thank Gennaro Chierchia, Veneeta Dayal, Danny Fox, the reviewers of SALT 29, and the audiences at ILLC, MIT, Rutgers, and SALT 29. All errors are mine. 
Getting quantifying-into questions uniformly

henceforth) (Groenendijk \& Stokhof 1984; Chierchia 1993). A general paraphrase for QIQ-readings of questions is given in (2), where 'Det' stands for a determiner. In particular, for questions with an existential quantifier (called $\exists$-questions), their QIQ-readings have a choice flavor. For example, (3a) asks to choose one/two kids and specify the book they read. Questions with a decreasing quantifier do not have QIQ-readings. For example, (3b) cannot be responded by silence.

(2) Which book did Det-kid(s) read?

$\approx$ 'For Det-kid(s), [tell me] which book did they read?'

a. Which book did one/two of the kids read?

Choice: 'For one/two of the kids, [tell me] which book did they read?'

b. Which book did no kid read?

\#'For no kid, [tell me] which book did they read?'

The other way is to treat pair-list questions uniformly. The multi-wh question (4) also has a reading that requests to specify a list of kid-book pairs. Accounts adopting this line of thinking either use the same LF to compose the $\forall$-question (1) and the multi-wh questions (4) (Engdahl 1980, 1986; Dayal 1996, 2018) or at least assign these two questions with the same root denotation (Fox 2012a,b).

(4) Which kid read which book?

a. Single-pair: 'Which unique kid- $x$-to-book- $y$ pair is s.t. $x$ read $y$ ?'

b. Pair-list: 'What are the kid- $x$-to-book- $y$ pairs s.t. $x$ read only $y$ ?'

In sum, it is controversial whether we should treat questions with QIQ-readings uniformly or questions with pair-list readings uniformly. This paper argues for the former option and provides a compositional analysis to derive QIQ-readings. The rest of this paper is organized as follows. Section 2 presents evidence for the view of unifying the composition of QIQ-questions. Section 3 reviews two approaches to composing $\forall$-questions, including the function-based approach of Dayal 1996 and the family-of-question approach of Fox 2012b. Section 4 introduces a hybrid categorial approach (Xiang 2016, 2019), which I will use as a general framework for question composition. Section 5 presents a uniform definition and a compositional analysis for questions with QIQ-readings. Section 6 uses the presented account to account for the quantificational variability effects in embeddings of $\forall$-questions. Section 7 concludes.

\section{Arguments for unifying QIQ-readings}

This section argues that $\forall$-questions with pair-list readings should be composed uniformly as other questions with QIQ-readings, not the corresponding multi-wh 
questions with pair-list readings. On the one hand, when taking pair-list readings, $\forall$-questions are subject to domain exhaustivity while their multi- $w h$ counterparts are not (\$2.1); therefore, the pair-list readings of these two types of questions must be defined and derived differently. On the other hand, questions with QIQ-readings should have a uniform LF: these questions are subject to similar syntactic constraints such as subject-object/adjunct asymmetries, and moreover, the distribution of QIQreadings is preserved in questions with a coordination of quantifiers (\$2.2).

\subsection{Domain exhaustivity}

It is dominantly claimed that pair-list readings of multi-wh-questions and $\forall$-questions are both subject to a domain exhaustivity condition, which says that every member of the set quantified over by the $w h-/ \nabla$-subject must be paired with a member of the set quantified over by wh-object (Dayal 2002). In (1) and (4), for instance, domain exhaustivity requires that every kid read a (possibly different) book. Moreover, since the object-wh is singular-marked, the two questions are also subject to point-wise uniqueness, which requires that each kid read at most one book. The point-wise uniqueness effect is easy to detect, but the domain exhaustivity effect is quite obscure. For example, in "which kid read which book?", it is unclear which set of kids is quantified over by the subject-wh. Domain exhaustivity would be trivial if this quantification domain is a subset of kids who did read a book. To remove this confound, Fox (2012a) uses the examples in (5), where every wh-phrase has an explicit quantification domain. Fox claims that (5b) rejects a pair-list reading (in contrast to (5a)), arguing that this reading is rejected because the domain exhaustivity condition presupposed in a pair-list reading is contextually infelicitous — pairing 4 kids with 3 chairs yields that there will be multiple kids sitting on the same chair.

a. Guess which one of the 3 kids will sit on which one of the 4 chairs.

b. Guess which one of the 4 kids will sit on which one of the 3 chairs.

In contrast to the dominant view, I argue that multi-wh questions are not subject to domain exhaustivity, unlike $\forall$-questions. First, multi-wh questions with pair-list readings can be used in contexts where domain exhaustivity is violated. In (6), the sentence copied from (5b) is fully acceptable and must be interpreted as pair-list.

(6) (Context: 4 kids are playing Musical Chairs and are competing for 3 chairs.) "Guess which one of the 4 kids will sit on which one of the 3 chairs." \& Each of the 4 kids will sit on one of the 3 chairs.

The game rules of Musical Chairs yield two conditions: (i) one of the 4 kids will not sit on any of the 3 chairs, and (ii) the rest 3 kids each will sit on a different chair. Condition (ii) ensures that the embedded multi-wh question has a pair-list reading, 
not a single-pair reading. Condition (i) contradicts the domain exhaustivity inference that each of the kids will sit on one of the chairs. If multi-wh questions with pair-list readings presuppose domain exhaustivity, (6) would suffer a presupposition failure and would be infelicitous in the given context, contra fact.

Second, in contrast, $\forall$-questions with pair-list readings cannot be used in contexts where domain exhaustivity is violated. In the context in (7), the quantification domain of the subject-wh/quantifier is greatly larger than that of the object-wh. The multi-wh question (7a) is fully acceptable, but the $\forall$-question (7b) isn't: (7b) presupposes that each candidate will get one of the jobs, contra context.

(Context: 100 candidates are competing for 3 job openings.)

a. $\sqrt{ }$ "Guess which candidate will get which job."

b. \#"Guess which job will every candidate get."

One might suggest that the domain exhaustivity condition of a multi-wh question can be associated with any of the wh-phrases, including the object-wh. For example, in (6) and (7), it could be the case that domain exhaustivity requires every chair and every job to be be taken by a kid and a candidate, respectively. However, this possibility is also ruled out: a multi- $w h$ question can be uttered in a context where neither type of domain exhaustivity is satisfied. For example, the sentence (8) is felicitous, and it doesn't imply domain exhaustivity relative to boys or to girls.

(8) (Context: 4 boys and 4 girls will form 4 boy-girl pairs to perform in a dance competition, but only 2 of the pairs will get into the final round.)

"Guess which one of the 4 boys will dance with which one of the 4 girls in the final round."

y $\rightarrow$ Each of the 4 boys will dance with one of the 4 girls in the final round.

\& $\rightarrow$ Each of the 4 girls will dance with one of the 4 boys in the final round.

In sum, pair-list readings of $\forall$-questions are subject to domain exhaustivity, while pair-list readings of multi-wh questions are not. Hence, these two pair-list questions should be defined and composed differently.

\subsection{Distributing QiQ-readings}

The distribution of QIQ-readings uniformly exhibits a subject-object/adjunct asymmetry (Chierchia 1991, 1993). As seen in (9) and (10), pair-list and choice readings are available if the non-wh quantifier serves as the subject and the $w h$-item serves as the object, and otherwise unavailable. The subject-adjunct asymmetry is analogous.
a. Which candidate did everyone vote for?
$\sqrt{ }$ pair-list
b. Which voter voted for every candidate?
$\times$ pair-list 
(10) a. Which candidate did one of the students vote for?

$\sqrt{ }$ choice

b. Which student voted for one of the candidates?

?choice

The similar distribution of pair-list readings and choice readings suggests that these readings are derived via a similar procedure in questions with a quantifier.

The above conjecture is further supported by the interpretations of questions with a coordination of quantifiers. In (11a) where the subject is a conjunction of a $\forall$ - and an $\exists$-quantifier, the pair-list reading associated with the $\forall$-quantifier and the choice reading associated with the $\exists$-quantifier are both preserved. In contrast, as decreasing quantifiers do not license QIQ-readings (recall (3b)), coordinating a $\forall / \exists$-quantifier with a decreasing quantifier blocks the QIQ-reading, as in (11b-c).

(11) a. Which book did [each of the boys and one of the girls] read? ( $\sqrt{ } \mathrm{QIQ})$

b. Which book did [each of the boys and none of the teachers] read? ( $\times$ QIQ)

c. Which book did [one of the girls and none of the teachers] read? $(\times \mathrm{QIQ})$

\section{Two existing accounts}

There is a rich literature on composing questions with quantifiers. For recent overviews, see Xiang 2016: ch. 6, Dayal 2018: ch. 4, and Ciardelli \& Roelofsen 2018. This section reviews only the accounts by Dayal $(1996,2018)$ and Fox $(2012 b)$, because they successfully predict the domain exhaustivity and point-wise uniqueness effects in singular-marked $\forall$-questions, and because the proposed account will take ingredients from these two accounts.

\subsection{Function-based approach (Dayal 1996, 2018)}

Function-based approaches to question composition assume that $\forall$-questions and multi-wh-questions with pair-list readings involve a functional dependency between the higher $\forall / w h$-phrase and the lower $w h$-phrase (Engdahl 1980, 1986; Groenendijk \& Stokhof 1984; Chierchia 1993). Adopting this view, Dayal (1996, 2018) assumes that the two questions in (12) both denote a set of conjunctive propositions, and that each of the contained conjunctive propositions specifies a Skolem function $f$ from the quantification domain of the $\forall / w h$-subject (kid@) to the quantification domain of the wh-object (book@). ${ }^{1}$ This denotation yields domain exhaustivity since the function $f$ takes the set of atomic kids as its domain.

(12) Which book did every kid read?/ Which kid read which book? (There are two relevant kids, $k_{1} k_{2}$, and two relevant books, $b_{1} b_{2}$.)

1 '@' stands for the actual world. For simplicity, here and henceforth, NP-restrictors in $w h$-phrases and generalized quantifiers are interpreted relative to the actual world. 
Getting quantifying-into questions uniformly

$$
\begin{aligned}
& \llbracket Q_{\forall} \rrbracket=\llbracket Q_{\text {multi-wh }} \rrbracket=\left\{\bigcap\left\{\hat{r} \operatorname{read}(x, \mathbf{f}(x)) \mid \operatorname{kid}_{@}(x)\right\} \mid \mathbf{f} \in\left[\operatorname{kid}_{0} \rightarrow \text { book@ }\right]\right\} \\
& =\left\{\begin{array}{c}
\hat{\operatorname{read}}\left(k_{1}, b_{1}\right) \cap \wedge \operatorname{read}\left(k_{2}, b_{1}\right) \\
\hat{\operatorname{read}}\left(k_{1}, b_{1}\right) \cap \cap^{\wedge} \operatorname{read}\left(k_{2}, b_{2}\right) \\
\hat{\operatorname{read}}\left(k_{1}, b_{2}\right) \cap^{\wedge} \wedge a d\left(k_{2}, b_{1}\right) \\
\hat{\operatorname{read}}\left(k_{1}, b_{2}\right) \cap^{\wedge} \operatorname{read}\left(k_{2}, b_{2}\right)
\end{array}\right\}
\end{aligned}
$$

Both questions in (12) are composed via the LF in (13). (For details and a review of this compositional analysis, see Xiang 2016: §5.3.1 and §6.2.3.) The sets that the $\forall / w h$-phrases range over are extracted by type-shifting operations and are passed to fill the range and the domain arguments of the functional $\mathrm{C}$ head.

$$
\text { [which-book } \left.{ }_{i}\left[\text { which/every-kid }{ }_{j}\left[\mathrm{C}^{\prime} \mathrm{C}_{\text {func }}^{0}\left[\mathrm{IP} t_{j} \operatorname{read} t_{i}^{j}\right]\right]\right]\right]
$$

Finally, Dayal defines an answerhood-operator that presupposes the existence of the strongest true answer. The strongest true answer to a question is the true proposition in the Hamblin set that entails all the true propositions in this set.

$$
\operatorname{ANS}_{\text {Dayal }}(w)(Q)=\imath p[w \in p \in Q \wedge \forall q[w \in q \in Q \rightarrow p \subseteq q]]
$$

When the NP-complement of the object- $w h$ is singular, ranging over only atomic items, the presupposition of $\mathrm{ANS}_{\text {Dayal }}$ entails point-wise uniqueness. For example, if $k_{1}$ read only $b_{1}$ but $k_{2}$ read both $b_{1} b_{2}$, then the top two propositions in the Hamblin set $Q$ in (12) are both true but neither is stronger than the other.

Dayal's account successfully predicts domain exhaustivity and point-wise uniqueness effects in singular-marked $\forall$-questions. It also manages to keep the semantic type of questions low (i.e., single/double-wh questions and $\forall$-questions are uniformly of type $\langle s t, t\rangle$ ), which leaves space for tackling constructions that are more complex.

However, this account also faces a couple of problems. This paper considers only the problems related to empirical predictions. First, treating $\forall$-questions and multi-wh-questions uniformly, this account cannot explain their contrast with respect to domain exhaustivity. Second, this account has difficulties in accounting for the quantificational variability $(\mathrm{QV})$ effects in embeddings of pair-list questions (Lahiri 2002). For example, the question-embedding sentence (15) implies a QV inference, which can be paraphrased as if the matrix quantity adverbial mostly quantifies over a set of atomic propositions. However, these atomic propositions cannot be retrieved from the question denotation assumed in (12), because the conjuncts of a conjunctive proposition cannot be extracted from this proposition.

(15) Jenny mostly knows which book every kid read.

$\rightsquigarrow$ 'For most propositions $p$ of the form 'kid- $x$-read-book- $y$ ', J knows $p$.'

Third, this account does not extend to $\exists$-questions. To avoid over-generating pairlist readings for $\exists$-questions (see more of this motivation in section 5.2.2), Dayal stipulates that the quantification domain of a non-interrogative quantifier must be 
extracted as the unique minimal witness set of this quantifier. ${ }^{2}$ With this stipulation, if a question with a non- $\forall$-quantifier is assigned with the LF in (13), the composition would crash - either the quantifier has multiple minimal witness sets, or its minimal witness set is the empty set. Although this assumption avoids over-generating pairlist readings in non- $\forall$-questions, it leaves choice readings of $\exists$-questions unexplained.

\subsection{Family-of-question approach (Fox 2012b)}

Fox (2012a,b) proposes a family-of-questions approach to composing complex questions, which regards pair-list questions as denoting sets of sub-questions. (This idea is also defended for or adopted by Hagstrom 1998, Fox 2012a, Nicolae 2013, Kotek 2014, a.o.). As exemplified in (16), if a simple single-wh question denotes a set of propositions, a family of questions denotes a set of sets of propositions.

Which book did every kid read?/ Which kid read which book?

(There are two relevant kids $k_{1} k_{2}$ and two relevant books $b_{1} b_{2}$.)

$$
\begin{aligned}
\llbracket Q_{\forall \rrbracket} \rrbracket \llbracket \llbracket Q_{\text {multi-wh }} \rrbracket & =\{\llbracket \text { which book did } x \operatorname{read} ? \rrbracket \mid x \in \text { kid } @\} \\
& =\{\{\hat{r} \operatorname{read}(x, y)|y \in \operatorname{book} @| x \in \text { kid } @\} \\
& =\left\{\begin{array}{l}
\left\{\hat{r} \operatorname{read}\left(k_{1}, b_{1}\right), \hat{r} \operatorname{read}\left(k_{1}, b_{2}\right)\right\} \\
\left\{\hat{r e a d}\left(k_{2}, b_{1}\right), \hat{\operatorname{read}}\left(k_{2}, b_{2}\right)\right\}
\end{array}\right\}
\end{aligned}
$$

Fox composes the two pair-list questions via different LFs that yield the same root denotation. The LF of the $\forall$-question is given in (17a), read as 'the minimal set

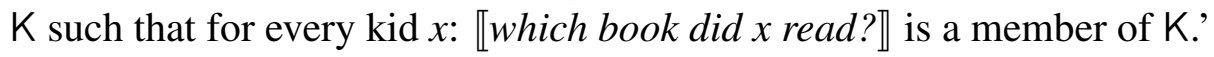

[MIN $\lambda \mathrm{K}[[\mathrm{DP}$ every kid] $\lambda x[\mathrm{~K}[\mathrm{CP} 1$ which book did $x$ read] $]]]$

The most important operations involved in forming this LF are quantifying-into a predication condition and moving a minimizer (MIN) (a la Pafel 1999; Preuss 2001).

$$
\llbracket \mathrm{MIN} \rrbracket=\lambda \alpha . \imath \mathrm{K}\left[\mathrm{K} \in \alpha \wedge \forall \mathrm{K}^{\prime} \in \alpha\left[\mathrm{K}^{\prime} \subseteq \mathrm{K}\right]\right]
$$

(For a set of sets $\alpha$ : the unique set in $\alpha$ that is a superset of every set in $\alpha$.)

In (17), first, the $\forall$-subject undergoes QR and quantifies into a predication condition between an null operator $\mathrm{K}$ and an open $w h$-question, yielding a universal predication

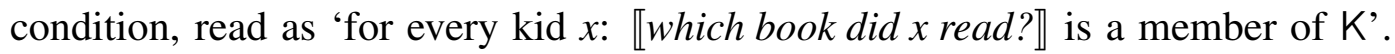
Next, MIN binds the $\mathrm{K}$ variable across the $\forall$-subject, returning the unique minimal $\mathrm{K}$ set that satisfies the universal predication condition. This minimal set is simply the set consisting of all the sub-questions of the form which book did kid-x read?.

2 Witness sets are defined as follows (Barwise \& Cooper 1981): (i) a generalized quantifier $\pi$ lives on a set $B$ iff $\pi(C) \Leftrightarrow \pi(C \cap B)$ for any set $C$; (ii) if $\pi$ lives on $B$, then $A$ is a witness set of $\pi$ iff $A \subseteq B$ and $\pi(A)$. For example, given a discourse domain including three kids $a b c$, the universal quantifier every kid has a unique minimal witness set $\{a, b, c\}$, while the existential quantifier one of the kids has three minimal witness sets $\{a\},\{b\}$, and $\{c\}$, each of which consists of one kid. 
Further, Fox assumes that answering a family of sub-questions means answering each of the contained sub-question. As such, answerhood is applied point-wise. As recursively defined in (19), the point-wise answerhood-operator imposes $\mathrm{ANS}_{\text {Dayal }}$ to each sub-question and returns the conjunction of the derived propositional answers of the sub-questions, yielding domain exhaustivity and point-wise uniqueness.

$$
\mathrm{ANS}_{\mathrm{PW}}=\lambda w \lambda Q \cdot \begin{cases}\operatorname{ANS}_{\text {Dayal }}(w)(Q) & Q \text { is of type }\langle s t, t\rangle \\ \bigcap\left\{\operatorname{ANS}_{\mathrm{PW}}(w)(\alpha) \mid \alpha \in Q\right\} & \text { otherwise }\end{cases}
$$

Fox's account has two advantages over Dayal's account. First, it manages to treat the $\forall$-subject a regular generalized quantifier — in composition, every kid combines with a predicate of type $\langle e, t\rangle$ denoted by its sister node. There is no need to stipulate a type-shifting operation to extract the quantification domain. Second, assigning $\forall$-questions and multi-wh- questions with non-flat semantics (i.e., as sets of sets), this account can capture the QV effects in the embeddings in (15). The QV inference can be defined by making MOST quantify over a set of sub-questions, read as follows: 'for most sub-questions Q of the form 'which book did kid- $x$ read', Jenny knows Q.'

However, the same as Dayal's account, Fox's account defines $\forall$-questions equivalent to multi-wh-questions and therefore it cannot explain their contrast with respect to domain exhaustivity. Moreover, this account also does not extend to $\exists$-questions. Fox uses the MIN-operator to get the unique minimal $\mathrm{K}$ set that satisfies a quantified predication relation, which exists only if the predication is universally quantified.

\section{Framework: A hybrid categorial approach of questions}

My general treatment of question question follows the hybrid categorial approach developed in Xiang 2016 and Xiang 2019. For the issues concerned in this paper, this approach is advantageous in getting QV effects in embeddings of $\forall$-questions (compared with proposition-based accounts such as Hamblin-Karttunen Semantics) and avoiding type-mismatch in composing functional readings (compared with traditional categorial approaches). Note that, however, assumptions on the structure of the question nucleus of a QIQ-question will be independent from this framework.

The hybrid categorial approach has three main ingredients. First, matrix and embedded questions uniformly denote functions from short answers to propositional answers, called "topical properties". For example, the question in (20) denotes a function that maps each atomic student $x$ to the proposition that $x$ came. As such, short answers are extractable from question denotations as meanings in the property domain. This assumption is basic in any categorial approach to question composition. It will be important in accounting for QV inferences (§6).

(20) Which student came? Jenny. 

a. $Q=\lambda x_{e}$ : student $@(x)$. ${ }^{\wedge} \operatorname{came}(x)$
b. $Q(j)=\operatorname{student}_{@}(j)$. ^came $(j)$

Second, in the compositional derivation, a $w h$-phrase is an $\exists$-quantifier in lexicon but is type-shifted into a function domain restrictor via the application of a BEDOMoperator. For any $\exists$-quantifier $\pi, \operatorname{BE}(\pi)$ is the set that $\pi$ ranges over (Partee 1986), and then $\operatorname{BEDOM}(\pi)$ is a function domain restrictor which combines with a function $\theta$ and returns the function that is similar to $\theta$ but undefined for items not in $\operatorname{BE}(\pi)$.

$$
\operatorname{BEDom}(\pi)=\lambda \theta_{\tau} \cdot l P_{\tau}\left[\begin{array}{r}
{[\operatorname{Dom}(P)=\operatorname{Dom}(\theta) \cap \operatorname{BE}(\pi)]} \\
\wedge \forall \alpha \in \operatorname{Dom}(P)[P(\alpha)=\theta(\alpha)]
\end{array}\right]
$$

For example, (22) is the LF for the question in (20). Here 'BEDOM (which student)' combines with the came-function defined for any individuals and returns the camefunction defined only for individuals that are atomic students.

$$
\left[{ }_{C P}[\mathrm{DP} \operatorname{BEDOM}(\text { which student })] \lambda x[\text { [IP } x \text { came }]\right]
$$

Crucially, $\operatorname{BEDom}(\pi)$ is type-flexible - it can combine with any function of an $\langle\sigma, \ldots\rangle$ type where $\sigma$ is the type of an element in $\operatorname{BE}(\pi)$. The type-flexibility makes it to compose a question regardless of the number of $w h$-phrases and regardless of whether the domain of the topical property is a set of individuals or functions.

Last, the quantification domain of a wh-phrase ' which-A' is polymorphic - it consists of not only elements in $A$ but also Skolem functions to $A$.

$$
\begin{aligned}
\llbracket \text { which }-A \rrbracket=\lambda P . \exists \alpha \in(A \cup\{\mathbf{f} \mid \operatorname{Range}(\mathbf{f}) \subseteq A\})[P(\alpha)] \\
\text { where Range }(\mathbf{f}) \subseteq A \text { iff } \forall x \in \operatorname{Dom}(\mathbf{f})[\mathbf{f}(x) \in A]
\end{aligned}
$$

As such, a $w h$-question has an individual reading if the $w h$-phrase binds an individual trace, as in (24a), and a functional reading if it binds a functional trace, as in (24b). ${ }^{3}$

3 In (24b), the functional $w h$-trace has an individual argument $x$ bound by the subject-quantifier every kid. This functional dependency can be achieved in two ways. One way is to interpret the $w h$-trace as a complex functional trace (Groenendijk \& Stokhof 1984; Engdahl 1986; Chierchia 1993; Dayal 1996, 2018): the $w h$-trace $t_{i}^{j}$ carries two indices, including a functional index $i$ which is bound by the $w h$-phrase and interpreted as a functional variable of type $\langle e, e\rangle$, and an argument index $j$ which bound by the subject-quantifier and interpreted as an individual variable of type $e$.

$$
\left.\left[\text { Which-book }{ }_{i} \ldots \text { [every-kid }{ }_{j}\left[t_{j} \operatorname{read} t_{i}^{j}\right]\right]\right]
$$

Another way uses combinatory rules as in Variable-free Semantics (Jacobson 1994, 1995, 1999). Jacobson interprets a functional $w h$-trace as an identity function over Skolem functions, namely: $\llbracket t \rrbracket=$ $\lambda f_{\langle e, e\rangle} . f$. Then she defines a locally applied $\mathbf{z}$-rule which can close off the functional dependency between the subject and the object of a transitive verb. This derivation does not involve any index. I use the former approach for compatibility with assumed formal theory. However, the latter approach is more advantageous in cases where a complex functional trace cannot be properly created by wh-movement. 
Getting quantifying-into questions uniformly

Which book did every kid read?

a. Individual $\quad \mathrm{Q}=\lambda x_{e}$ : $\operatorname{book}_{@}(x) .{ }^{\wedge} \forall x[\operatorname{kid}(x) \rightarrow \operatorname{read}(x, y)]$ [CP [DPBEDOM(which student)] $\lambda y_{e}$ [IP every kid read $\left.\left.y\right]\right]$

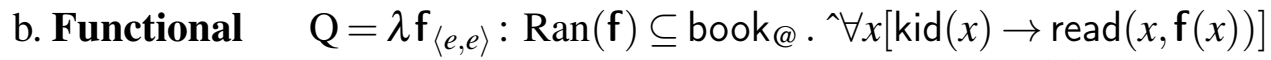
$\left[\mathrm{CP}\left[{ }_{\mathrm{DP}} \mathrm{BEDOM}(\right.\right.$ which student $\left.)\right] \lambda \mathbf{f}_{\langle e, e\rangle}\left[\mathrm{IP}\right.$ every $\left.\left.\operatorname{kid}_{x} \operatorname{read} \mathbf{f}(x)\right]\right]$

\section{Proposal}

I analyze QIQ-readings as special functional readings and derive them uniformly by employing quantification-into predication and moving an existential minimizer.

\subsection{Question denotation}

In line with function-based approaches to composing questions with quantifiers (Engdahl 1980, 1986; Groenendijk \& Stokhof 1984; Chierchia 1993; Dayal 1996, 2018; Sharvit \& Kang 2017; a.o.), I propose that QIQ-reading is a special functional reading. Namely, the $w h$-phrase binds a functional trace, and the yielded question denotation is a topical property of Skolem functions, as defined uniformly in (25).

(25) Which book did Det-kid(s) read?

$$
\begin{aligned}
Q=\lambda \mathbf{f}_{\langle e, e\rangle}: \operatorname{Range}(\mathbf{f}) \subseteq \text { book } @ & \wedge \text { Det-kid }(\operatorname{Dom}(\mathbf{f})) . \\
& \bigcap E_{\mathrm{MIN}}\left\{\mathrm{K} \mid \operatorname{Det}-k i d\left(\lambda x . \mathrm{K}\left({ }^{\wedge} x \text {-read-f }(\mathrm{x})\right)\right)\right\}
\end{aligned}
$$

This topical property is defined for Skolem functions that map Det-kid to atomic books. The output is the conjunction of a proposition set that ranges over exactly Detkid. I will go through the compositional derivation of this denotation in section 5.2. The following only writes out the immediate predicted denotations of $\forall$-questions. If Det is a universal determiner like every or each, the topical property is as follows (the restriction on the range of $f$ is omitted):

$$
\begin{aligned}
Q_{\forall} & =\lambda \mathbf{f}: \ldots \wedge \forall-\text { kid }_{@}(\operatorname{Dom}(\mathbf{f})) \cdot \bigcap E_{\mathrm{MIN}}\left\{\mathrm{K} \mid \forall-\text { kid }_{0}\left(\lambda x \cdot \mathrm{K}\left({ }^{\wedge} x \text {-read-f }(\mathrm{x})\right)\right)\right\} \\
& =\lambda \mathbf{f}: \ldots \wedge \forall-\mathrm{kid}_{@}(\operatorname{Dom}(\mathbf{f})) \cdot \bigcap\left\{{ }^{\wedge} \operatorname{read}(x, \mathbf{f}(x)) \mid \mathrm{kid}_{@}(x)\right\}
\end{aligned}
$$

Here the input function $\mathbf{f}$ is one that pairs every kid with a book, and the output is the conjunction of the set that consists of every proposition of the form ^ kid- $x$-read- $f(x)$, which is simply the set $\left\{{ }^{\wedge} \operatorname{read}(x, \mathbf{f}(x)) \mid \operatorname{kid}_{@}(x)\right\}$. Crucially, domain exhaustivity is predicted since the input $\mathbf{f}$ must be defined for every kid.

\subsection{Composition}

The question denotation in (25) is compositionally derived via the LF in Figure 1. In particular, the restriction on the range of the input Skolem functions (i.e., that $f$ 


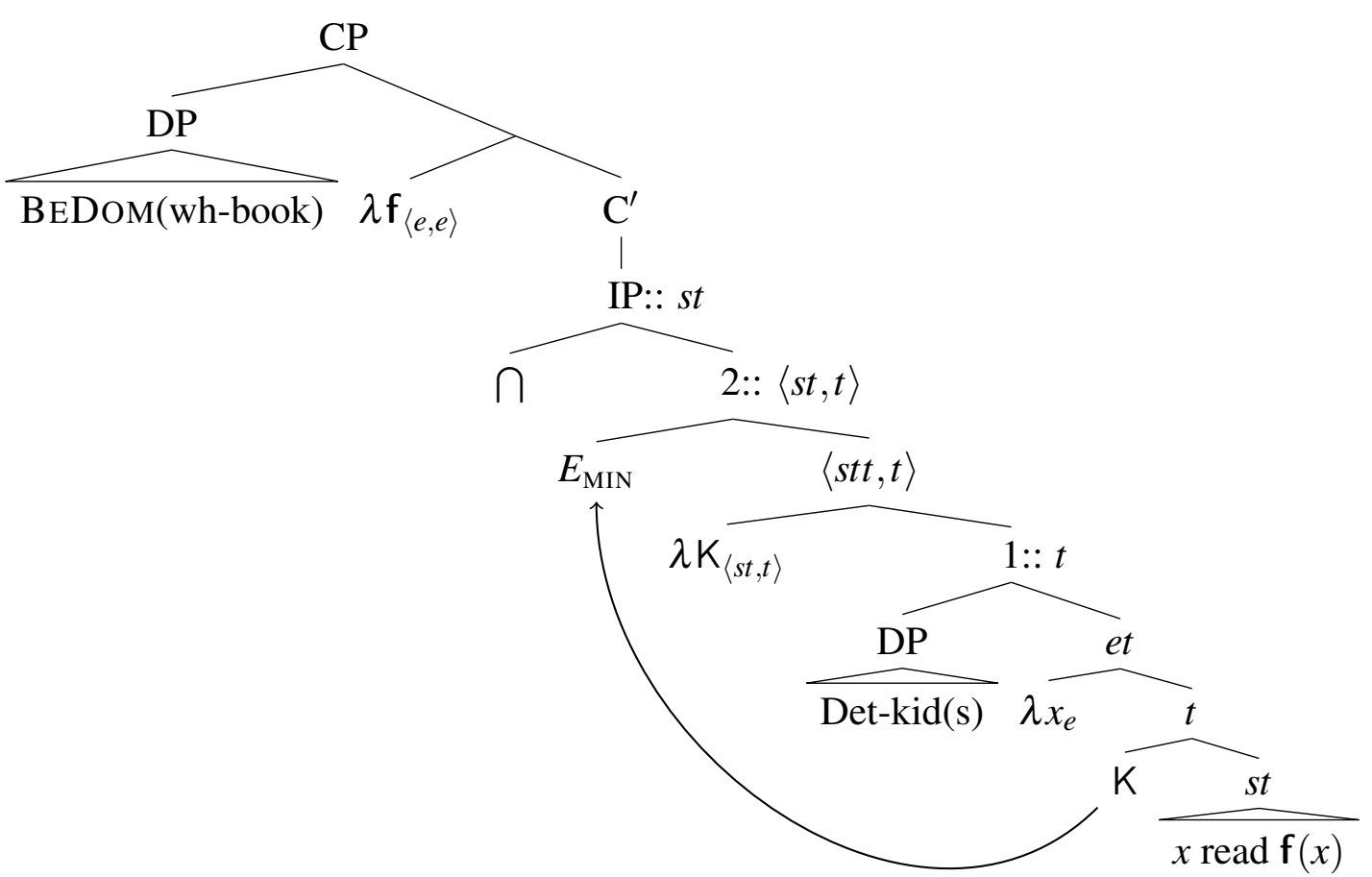

Figure 1 LF for deriving the QIQ-reading of 'which book did Det-kid(s) read?'

ranges to atomic books) is supplied by the wh-phrase, and all the rest (including the restriction on the domain of the input Skolem function and the output proposition) are from the question nucleus (viz., IP).

On the one hand, the same as in the LF of a basic functional reading (see (24b)), the wh-object $\mathrm{BEDOM}($ wh-book $)$ moves to [Spec, $\mathrm{CP}$ ] and leaves a functional trace, and the subject-quantifier Det-kid(s) undertakes QR and binds the argument variable of this functional trace. These movements and bindings yield functional dependency from the subject-quantifier to the wh-object, making the reading "functional".

On the other hand, different from the LF of a basic functional reading, here the nucleus involves two covert operations, namely, predicating over an open sentence and moving a minimizer. These operations are similar to what Fox (2012b) assumes in (17), but their nuances yield desirable consequences in accounting for domain exhaustivity in $\forall$-questions and unifying the derivation of QIQ-readings. First, the predication condition assumed this the presented analysis is applied to an open-sentence that expresses a functional dependency relation between a functional variable $\mathrm{f}$ and an individual variable $x$. As will be seen in section 5.2.1, the bindings of these two variables contribute to the derivation of domain exhaustivity. Second, the choice minimizer $E_{\mathrm{MIN}}$-operator, as defined in (27), is semantically weaker than 
Getting quantifying-into questions uniformly

the Pafel-Fox MIN-operator and does not presuppose uniqueness. ${ }^{4}$ Replacing MIN with $E_{\mathrm{MIN}}$ makes the analysis extendable to $\exists$-questions (section 5.2.2).

$\llbracket E_{\mathrm{MIN}} \rrbracket=\lambda \alpha \cdot f_{\mathrm{CH}}\left(\left\{\mathrm{K} \mid \mathrm{K} \in \alpha \wedge \forall \mathrm{K}^{\prime}\left[\mathrm{K} \not \subset \mathrm{K}^{\prime}\right]\right\}\right)$

(For a set of sets $\alpha$ : one of the sets in $\alpha$ that are not proper subsets of any set in $\alpha$. [ $f_{\mathrm{CH}}$ stands for a choice function.])

Let $a$ and $b$ be two distinct entities, $A=\{\varnothing,\{a\},\{b\}\}$, and $B=\{\{a\},\{b\}\}$.

a. $\llbracket \operatorname{MIN} \rrbracket(A)=\llbracket E_{\mathrm{MIN}} \rrbracket(A)=\varnothing$;

b. $\llbracket \mathrm{MIN} \rrbracket(B)$ is undefined; $\llbracket E_{\mathrm{MIN}} \rrbracket(B)$ has two possible values: $\{a\}$ or $\{b\}$.

\subsubsection{Composing $\forall$-questions}

This section presents the details of composing the $\forall$-question (29). I divide the LF into three parts — node 1 , the rest of node 2 , and the rest of the full CP.

(29) Which book did every kid read?

[CP BEDOM(wh-book) $\lambda \mathrm{f}\left[{ }_{\mathrm{IP}} \bigcap\left[{ }_{2} E_{\mathrm{MIN}} \lambda \mathrm{K}[1\right.\right.$ every-kid $\lambda x[\mathrm{~K}(x$-read-f $\left.\left.(x))]]\right]\right]$

a. $\llbracket 1 \rrbracket=\forall x \in \mathrm{kid}_{\mathrm{a}}\left[\mathrm{K}\left({ }^{\wedge} x\right.\right.$-read-f $\left.\left.\mathrm{f}(x)\right)\right]$

b. $\lambda \mathrm{K} . \llbracket 1 \rrbracket=\lambda \mathrm{K} . \forall x \in \mathrm{kid}_{@}[\hat{\imath} x$-read-f $(x) \in \mathrm{K}]$

$$
=\lambda \mathrm{K}: \forall x \in \operatorname{kid}_{@}[x \in \operatorname{Dom}(\mathbf{f})] \cdot\left\{\hat{x} x \text {-read-f }(x) \mid \operatorname{kid}_{@}(x)\right\} \subseteq \mathrm{K}
$$

c. $\llbracket 2 \rrbracket=\llbracket E_{\mathrm{MIN}} \rrbracket(\lambda \mathrm{K} . \llbracket 1 \rrbracket)=\forall x \in \operatorname{kid}_{@}[x \in \operatorname{Dom}(\mathbf{f})] \cdot\left\{{ }^{\wedge} x\right.$-read-f $\left.(x) \mid \operatorname{kid}_{@}(x)\right\}$

d. $\llbracket \mathrm{IP} \rrbracket=\forall x \in \operatorname{kid}_{@}[x \in \operatorname{Dom}(\mathbf{f})] \cdot \bigcap\left\{\hat{x}\right.$-read-f $\left.(x) \mid \operatorname{kid}_{@}(x)\right\}$

e. $\llbracket \mathrm{CP} \rrbracket=\lambda \mathbf{f}_{\langle e, e\rangle}: \operatorname{Range}(\mathbf{f}) \subseteq$ book $@ \wedge \wedge x \in \operatorname{kid}_{@}[x \in \operatorname{Dom}(\mathbf{f})]$.

$$
\bigcap\left\{{ }^{\wedge} x \text {-read-f }(x) \mid \text { kid }_{@}(x)\right\}
$$

Part 1 (node 1): Quantify-into the predication over an open sentence. First, an operator K combines with an open sentence $x$-read- $\mathrm{f}(x)$, yielding a simple predication condition that this open sentence is a member of $\mathrm{K}$. Next, the subject-quantifier Det$k i d(s)$ quantifies into this predication condition. If Det is the universal determiner every, we have a universal predication condition as in (29a). This part of composition, on the one hand, inherits the advantage of Fox's analysis that the subject-quantifier standardly combines with a predicate of type $\langle e, t\rangle$. On the other hand, since here the subject-quantifier also binds the argument variable of the functional trace, the quantifying-in operation also yields a presupposition that the Skolem function $f$ is defined for Det-kid(s). For example, the universal predication condition in (29a) is defined only if $f$ is defined for every kid. This presupposition projects over $\mathrm{CP}$, yielding domain exhaustivity for the $\forall$-question.

4 For readers who are familiar with Boolean Semantics, the $E_{\mathrm{MIN}}$-operator is basically the collectivity raising operator in Winter 2001. 


$$
\forall x \in \operatorname{kid} @\left[\left[\mathrm{~K}\left({ }^{\wedge} \text {-read- } \mathbf{f}(x)\right)\right] \text { is defined only if } \forall x \in \operatorname{kid}_{@}[x \in \operatorname{Dom}(\mathbf{f})]\right.
$$

Note that the derivation of domain exhaustivity crucially relies on the presence of a $\forall$-quantifier - the domain exhaustivity effect comes from quantifying-into a predication condition and especially the binding relation between a $\forall$-quantifier and the argument of the functional trace. Hence, in contrast to the family-of-questions approach by Fox (2012a,b), this analysis does not predict domain exhaustivity for questions without a $\forall$-quantifier. ${ }^{5}$

Part 2 (to node 2): Abstract the variable $\mathrm{K}$ and apply a choice minimizer $E_{\mathrm{MIN}}$. Abstracting the variable $K$ returns the set of the $K$ sets that satisfy the quantificational predication condition yielded by Node 1 . In the $\forall$-question, these are the sets that contain all the propositions of the form $\operatorname{kid}-x$-read-f $(x)$, as in (29b). At node 2, applying the minimizer $E_{\mathrm{MIN}}$ returns one of the eligible minimal $\mathrm{K}$ sets. There is only one minimal set that satisfy the universal quantification predication, namely, the set of the propositions of the form kid- $x$-read-f $f(x)$, as in (29c).

Part 3 (the rest): Apply a conjunctive closure and combine with the wh-phrase. The set of propositions denoted by node 2 is immediately closed by a $\bigcap$-closure, returning a conjunctive proposition. Following Dayal (2018), I consider this $\cap$ closure a function graph creator (FGC) and the yielded conjunctive proposition the graph of the function $\mathbf{f}$ (see also Xiang 2019 for the role of FGC in composing multi-wh questions with pair-list readings). Finally, BEDOM(which kid) binds the $f$ variable and restricts the range of $f$. The input of this topical property is a Skolem function that maps each kid to an atomic book, and the output is a conjunctive proposition describing the graph of this function.

To sum up, first, the QIQ-reading of the $\forall$-question (29) is subject to domain exhaustivity $([+\mathrm{D}-\mathrm{EXH}])$ because the universal predication condition (node 1$)$ is defined only if $\mathbf{f}$ is defined for every kid. Second, it expects a pair-list answer ([+PL]) because the yielded eligible minimal $\mathrm{K}$ set (node 2 ) is a non-singleton set ranging over multiple kids. Last, this reading does not have a choice flavor $([-\mathrm{CH}])$ because there is only one minimal eligible $\mathrm{K}$ set.

\subsubsection{Composing $\exists$-questions}

The composition of an $\exists$-question precedes analogously. Note 1 creates an existential predication condition over the open sentence $x$-read- $\mathbf{f}(x)$, as in (31a). Node 2 returns one of the minimal sets that satisfy this condition. Crucially, different from the case of the $\forall$-question, here there are multiple eligible minimal sets, each of which is a

5 For an analysis of composing multi-wh-questions using the assumed formal theory, see Xiang 2019. Basically, a multi-wh question has a similar semantics but does not presuppose domain exhaustivity. 
Getting quantifying-into questions uniformly

singleton set consisting of one proposition of the form kid- $x$-read- $f(x)$, as in (31b). (' $x=f_{\mathrm{CH}}($ kid $\left.@)\right)$ means that the kid $x$ is chosen by the choice function carried by the $E_{\mathrm{MIN}}$-operator.) Each such minimal set yields a possible question denotation, giving rise to a choice flavor. The rest steps precede the same as in the $\forall$-question.

Which book did one of the kids read?

$\left[{ }_{\mathrm{CP}} \operatorname{BEDOM}\left(\right.\right.$ wh-book) $\lambda \mathbf{f}\left[{ }_{\mathrm{IP}} \bigcap{ }_{2} E_{\mathrm{MIN}} \lambda \mathrm{K}\left[{ }_{1}\right.\right.$ one-kid $\lambda x[\mathrm{~K}(x$-read-f $\left.\left.\left.(x))]\right]\right]\right]$

a. $\llbracket 1 \rrbracket=\exists x \in \operatorname{kid}_{@}\left[\mathrm{~K}\left({ }^{\wedge} x\right.\right.$-read-f $\left.\left.\mathbf{f}(x)\right)\right]$

b. $\llbracket 2 \rrbracket=\llbracket E_{\mathrm{MIN}} \rrbracket(\lambda \mathrm{K} . \llbracket 1 \rrbracket)=\{\wedge$-read-f $(x)\}$, where $x=f_{\mathrm{CH}}\left(\operatorname{kid}_{@}\right)$

c. $\llbracket \mathrm{IP} \rrbracket=\wedge x$-read-f $(x)$, where $x=f_{\mathrm{CH}}\left(\right.$ kid $\left._{@}\right)$

d. $\llbracket \mathrm{CP} \rrbracket=\lambda \mathbf{f}_{\langle e, e\rangle}: \operatorname{Range}(\mathbf{f}) \subseteq$ book@ . $x$-read-f $(x)$, where $x=f_{\mathrm{CH}}($ kid $@ ~)$

I have shown that the QIQreading of the $\forall$-question (29) is [ $+\mathrm{D}-\mathrm{EXH},+\mathrm{PL},-\mathrm{CH}$ ], while here the QIQ-reading of the $\exists$-question $(31)$ is $[-\mathrm{D}$-EXH, $-\mathrm{PL},+\mathrm{CH}$ ]. This reading isn't subject to domain exhaustivity because the existential predication condition only requires $f$ to be defined for one kid. ${ }^{6}$ It is $[-\mathrm{PL}]$ because the minimal $\mathrm{K}$ sets satisfying the existential predication condition are all singleton sets. It has a choice flavor because there are multiple eligible minimal $\mathrm{K}$ sets.

The above discussion was on the question with the quantifier one of the kids. The rest of this section extends this analysis to other $\exists$-questions. As argued extensively in the literature (Srivastav 1991; Krifka 1991; Moltmann \& Szabolcsi 1994; Szabolcsi 1997; Beghelli 1997), pair-list readings are unavailable in any matrix question with a non- $\forall$-quantifier. For example, in the $\exists 2$-question (32), the seeming pair-list answer (32a) is actually a specification of the cumulative choice answer (32b).

(32) Who did two of the boys invite?

a. Andy invited Mary, and Billy invited Jenny.

b. Andy and Billy invited Mary and Jenny. In particular, Andy invited Mary and Billy invited Jenny.

The confound from cumulative answers will be removed once who is replaced with a singular-marked $w h$-phrase, which triggers uniqueness. In (33), distribution over uniqueness is only possible in (33a-b), where the subject is lexically distributive.

(33) I know that every student voted for a different candidate. Tell me ...

a. which candidate did every student vote for? $(\forall \gg \imath)$

b. which candidate did each of the students vote for?

$(\mathrm{EACH} \gg l)$

6 In (31d-e), there is no need to write out the domain condition that $\mathbf{f}$ must be defined for at least one kid, because this condition is entailed by the definedness condition of the output proposition: for any chosen kid $x$, the proposition $\wedge$-read- $\mathbf{f}(x)$ is defined only if $\mathbf{f}$ is defined for this $x$. 
c. \# which candidate did two of the students vote for? $(\exists 2 \gg E A C H \gg \imath)$

d. \# which candidate did most of the students vote for? (MOST $\gg E A C H \gg \imath)$

To avoid over-generating pair-list readings, existing works such as Dayal and Fox simply assume derivations of pair-list readings that would crash in questions with a non- $\forall$-quantifier. However, as seen in section 3 , these analyses come with an expense of not being able to account for choice readings of $\exists$-questions.

I propose that the determiner of the partitive quantifier two of the kids is not $\exists 2\left(=_{\text {def }} \lambda P_{\langle e, t\rangle} \lambda Q_{\langle e, t\rangle} \cdot|P \cap Q|=2\right)$ but rather $\exists\left(=_{\text {def }} \lambda P_{\langle e, t\rangle} \lambda Q_{\langle e, t\rangle} . P \cap Q \neq \varnothing\right)$; in other words, the numeral two is part of the restrictor. With this assumption, the quantifier two of the kids ranges over the set of entities that are pluralities of two kids and it denotes a set of sets that contain at least one of such plural entities. The composition of two of the kids precedes as follows. First, of combines with an entity denoted by the the-phrase and returns a set of subparts of this entity. Next, the numeral two, as a basic predicate restrictor, combines with a set of entities and returns a subset consisting of only the entities that have exactly two atomic subparts, as in (34c-d). Finally, a covert existential determiner $\varnothing_{\exists}$ combines with this set-denoting NumP and returns an existential generalized quantifier (Link 1987).

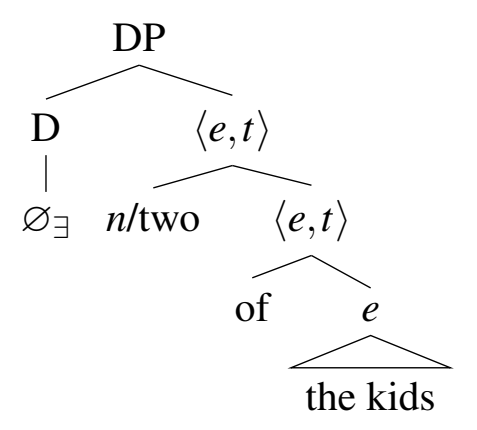

(34) If the discourse domain has three kids $a b c$ :

Now return to the composition of the $\exists 2$-question. 2-kids is abbreviated for the set of pluralities of two kids. The same as in (31c), here the eligible minimal $\mathrm{K}$ sets yielded from Node 2 are all singleton sets, as in (35c). Each of these sets consists one proposition of the form $x$-read- $\mathbf{f}(x)$ where $x$ is the plurality of two kids. As such, the derived QIQ-reading is [-PL].

(35) Which book did two of the kids read?

... [IP $\cap{ }_{2} E_{\mathrm{MIN}} \lambda \mathrm{K}[1$ two-of-the-kids $\lambda x[\mathrm{~K}(x$-read- $\left.\left.\mathrm{f}(x))]]\right]\right]$

a. $\llbracket 1 \rrbracket=\exists x \in 2$-kids@ $\left[\mathrm{K}\left({ }^{\wedge} x\right.\right.$-read-f $\left.\left.(x)\right)\right]$

b. $\llbracket 2 \rrbracket=\llbracket E_{\mathrm{MIN}} \rrbracket(\lambda \mathrm{K} \cdot \llbracket 1 \rrbracket)=\{\hat{\imath} x$-read-f $(x)\}$, where $x=f_{\mathrm{CH}}(2-$ kids@ $)$

This analysis also extends to matrix $\exists$-questions with a non-decreasing counting/comparative quantifier. In an $\exists$-question, as long as the $\exists$-quantifier is analyzed 
as being composed of a basic $\exists$-determiner and a numeral-modified restrictor (or say, the (bare or complex) numeral in the $\exists$-quantifier is treated as part of the restrictor, as in (36)), the minimal $\mathrm{K}$ set that satisfies the existential predication condition would be a singleton set, and the derived QIQ-reading would be [-PL].
a. $\llbracket$ exactly two kids $\rrbracket=\lambda P . \exists x[\# \operatorname{AT}(x)=2 \wedge P(x) \wedge$ kids@ $(x)]$
b. $\llbracket$ more than two kids $\rrbracket=\lambda P . \exists x[\# \mathrm{AT}(x)>2 \wedge P(x) \wedge$ kids@ $(x)]$

Strikingly, in contrast to matrix $\exists$-questions, embeddings of $\exists$-questions sometimes admit pair-list readings (Beghelli 1997; Szabolcsi 1997). For example, (37a) is felicitous even if every kid read a different book. I argue that this sentence has the LF in (37b): the $\exists$-quantifier moves over the embedding predicate, and its trace in the matrix clause is associated with a covert distributor EACH, which yields EACH $\gg l$.
a. Susi knows [which book two of the kids read].
b. [[ $\varnothing_{\exists}$ two-kids $\left.(x)\right] \lambda x[[x \mathrm{EACH}] \lambda y$ [Susi knows which book $y$ read $\left.]\right]$
c. $\exists x[x \in 2$-kids@ $\wedge \forall y \in \operatorname{AT}(x)[\llbracket$ Susi knows which book y read $\rrbracket]]$

In matrix $\exists$-questions, however, pair-list readings cannot be licensed by VP-each. In (38), the meaning contribution of each is just that the two chosen kids read the book separately, not that they read possibly a different book. The presented analysis explains the fact easily - VP-each is interpreted within the embedded sentence.

Which book did two of the kids each read? $(\exists 2 \gg \mathrm{EACH} \gg \imath)$
$\ldots$. IIP $\cap\left[{ }_{2} E_{\mathrm{MIN}} \lambda \mathrm{K}[1\right.$ two-of-the-kids $\lambda x[\mathrm{~K}(x$-each-read- $\left.\left.\mathrm{f}(x))]]\right]\right]$

\subsubsection{Composing questions with a decreasing quantifier}

Questions with a decreasing quantifier do not have QIQ-readings: (39a) cannot be responded by silence, and (39b) cannot be responded by naming a kid-book-pair.

a. Which book did \{no kid, none of the kids $\}$ read?

b. Which book did \{at most, less than $\}$ two kids read?

Following Hackl (2000), I treat a decreasing quantifier as being made up of a basic negative determiner $\neg \exists$ 'no' and a set-denoting restrictor, as in (40). The questions in (39) can thus be called " $\neg \exists$-questions".

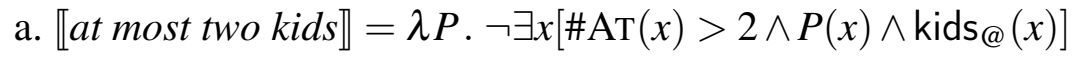

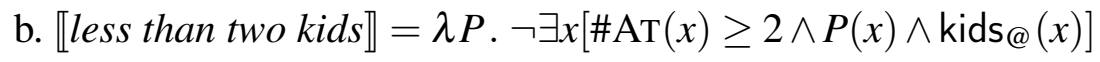

For any predication condition that expresses exclusion, the minimal $\mathrm{K}$ set that satisfies this condition is the empty set. (For example, the minimal set that contains no proposition of the form kid- $x$-read- $f(x)$ is the empty set $\varnothing$.) Thus, composing the 
above questions based on the LF in Figure 1 yields topical properties that map each input function to a contradiction. The main steps in composing (39a) are as follows:

$$
\begin{aligned}
& \ldots\left[\mathrm{IP} \cap\left[{ }_{2} E_{\mathrm{MIN}} \lambda \mathrm{K}\left[{ }_{1} \text { no-kid } \lambda x[\mathrm{~K}(x \text {-read-f }(x))]\right]\right]\right] \\
& \text { a. } \llbracket 1 \rrbracket=\neg \exists x \in \mathrm{kid}_{@}[\mathrm{~K}(\wedge x \text {-read-f } \mathrm{f}(x))] \\
& \text { b. } \llbracket 2 \rrbracket=\llbracket E_{\mathrm{MIN}} \rrbracket(\lambda \mathrm{K} . \llbracket 1 \rrbracket)=\varnothing
\end{aligned}
$$

\subsection{Interim summary}

To sum up, QIQ-readings are special functional readings - the object-wh leaves a functional trace, and the argument of this trace is bound by the subject-quantifier. The QIQ effect in questions like (42a) is derived by extracting the minimal $\mathrm{K}$ set that satisfies the quantificational predication condition (42b).

a. Which book did Det-kid(s) read?

b. DET-kids $\left(\lambda x \cdot \mathrm{K}\left({ }^{\wedge} x\right.\right.$-read-f $\left.\left.(x)\right)\right)$

This compositional analysis makes the following predictions. First, the QIQ-reading of (42a) is subject to domain exhaustivity ([+D-EXH]) if the quantificational predication condition ( $42 \mathrm{~b}$ ) presupposes that $\mathrm{f}$ is defined for every kid, as in $\forall$-questions. It rejects pair-list answers ([-PL]) if the extracted minimal $\mathrm{K}$ sets are all singletons, and has choice flavor $([+\mathrm{CH}])$ if there are multiple minimal $\mathrm{K}$ sets that satisfy the quantificational predication condition, as in $\exists$-questions. Moreover, QIQ-readings are unavailable if the extracted minimal $\mathrm{K}$ sets are empty sets, as in $\neg \exists$-questions.

\section{Quantificational variability effects}

In contrast to Dayal, this approach defines a $\forall$-question as a function from Skolem functions to propositions, from which we can get all the student-read-book pairs by retrieving the function that yields the strongest true answer of the $\forall$-question. This section only sketches out the basic idea. (For further details, see Xiang 2019.) The main take-away point is that QV inferences can be derived without treating pair-list questions as denoting families of sub-questions.

Let $\operatorname{ANS}^{S}(w)(Q)$ be the strongest true short answer of the question Q in $w$, and then the QV inference for a $\mathrm{Q}_{\forall}$-embedding sentence is schematized as in (43b). (See Cremers 2018 for a compositional derivation of this condition.)

(43) Jenny mostly knows which book every kid read.

a. $\operatorname{AT}(\mathbf{f})=\left\{\mathrm{f}^{\prime} \mid \mathrm{f}^{\prime} \subseteq \mathbf{f}\right.$ and $\bigoplus \operatorname{Dom}\left(\mathrm{f}^{\prime}\right)$ is atomic $\}$

b. $\lambda w . M O S T f^{\prime}\left[\mathrm{f}^{\prime} \in \operatorname{AT}\left(\operatorname{AnS}^{S}(w)(Q)\right)\right]\left[\operatorname{know}_{w}\left(j, \lambda w^{\prime} . \mathrm{f}^{\prime} \leq \operatorname{ANS}^{S}\left(w^{\prime}\right)(Q)\right)\right]$

(For most $\mathrm{f}^{\prime}$ that are atomic subparts of the strongest true short answer of $\mathrm{Q}$, Jenny knows that $\mathrm{f}^{\prime}$ is a subpart of the strongest true short answer of Q.) 


\begin{tabular}{|c|c|c|}
\hline & $\begin{array}{l}k_{1} \rightarrow b_{2} \\
k_{2} \rightarrow b_{2} \\
k_{3} \rightarrow b_{3}\end{array}$ & $\begin{array}{l}k_{1} \rightarrow b_{3} \\
k_{2} \rightarrow b_{2} \\
k_{3} \rightarrow b_{3}\end{array}$ \\
\hline $\begin{array}{l}k_{1} \rightarrow b_{1} \\
k_{2} \rightarrow b_{1} \\
k_{3} \rightarrow b_{3}\end{array}$ & $\begin{array}{l}k_{1} \rightarrow b_{1} \\
k_{2} \rightarrow b_{2} \\
k_{3} \rightarrow b_{3}\end{array}$ & $\begin{array}{l}k_{1} \rightarrow b_{1} \\
k_{2} \rightarrow b_{3} \\
k_{3} \rightarrow b_{3}\end{array}$ \\
\hline $\begin{array}{l}k_{1} \rightarrow b_{1} \\
k_{2} \rightarrow b_{2} \\
k_{3} \rightarrow b_{1}\end{array}$ & $\begin{array}{l}k_{1} \rightarrow b_{1} \\
k_{2} \rightarrow b_{2} \\
k_{3} \rightarrow b_{2}\end{array}$ & \\
\hline
\end{tabular}

Figure 2 Each cell is a set of worlds where the kid-read-book pairs are as the function enclosed.

The quantification domain of the matrix adverbial mostly is a set of atomic functions. The scope involves Jenny knowing a sub-divisive inference. ${ }^{7}$ If kid $k_{1} k_{2} k_{3}$ read book $b_{1} b_{2} b_{3}$ in $w$ respectively, then $\operatorname{ANS}^{S}(w)(Q)=\left[k_{1} \rightarrow b_{1}, k_{2} \rightarrow b_{2}, k_{3} \rightarrow b_{3}\right]$, and this sub-divisive inference is true iff in every world $w^{\prime}$ that is compatible with Jenny's belief, the strongest short answer of the embedded $\forall$-question in $w^{\prime}$ is one of the seven functions list in the partition in Figure 2. This inference is semantically equivalent to that Jenny correctly identifies most of the kid-read-book pairs.

\section{Conclusions}

In this paper, I firstly argued that pair-list readings of $\forall$-questions should be composed uniformly as other QIQ-readings and should be defined and derived differently from pair-list readings of multi-wh questions.

Next, I presented an analysis that derives QIQ-readings uniformly. I argue that QIQ-readings are special functional readings, namely, that the wh-object has a functional trace and that the subject-quantifier binds the argument variable of this functional $w h$-trace. The seeming QIQ effect is derived by extracting the minimal set that satisfies a quantificational predication relation. This analysis accounts for what questions admit QIQ-readings and whether their QIQ-readings are subject to domain exhaustivity $[ \pm \mathrm{D}-\mathrm{EXH}]$, admit pair-list answers $[ \pm \mathrm{PL}]$, and have a choice flavor $[ \pm \mathrm{CH}]$.

Finally, defining QIQ-readings as functions over Skolem functions, I showed that the presented analysis can also account for QV effects in embeddings of $\forall$-questions.

7 Note that here the scope of this QV inference cannot be written as $\operatorname{know}_{w}\left(j, Q\left(\mathrm{f}^{\prime}\right)\right)$. The topical property denoted by the question is only defined for functions that are defined for every kid, while here $\mathrm{f}^{\prime}$ is an atomic function and is only defined for one of the kids. 


\section{References}

Barwise, Jon \& Robin Cooper. 1981. Generalized quantifiers and natural language. Linguistics and Philosophy 4(2). 159-219.

Beghelli, Filippo. 1997. The syntax of distributivity and pair-list readings. In Ways of scope taking, 349-408. Springer.

Chierchia, Gennaro. 1991. Functional WH and weak crossover. In Proceedings of West Coast Conference on Formal Linguistics, vol. 10, 75-90.

Chierchia, Gennaro. 1993. Questions with quantifiers. Natural Language Semantics 1. 181-234.

Ciardelli, Ivano \& Floris Roelofsen. 2018. An inquisitive perspective on modals and quantifiers. Annual Review of Linguistics 4(1). 129-149.

Cremers, Alexandre. 2018. Plurality effects in an exhaustification-based theory of embedded questions. Natural Language Semantics 26(3). 193-251.

Dayal, Veneeta. 1996. Locality in Wh Quantification: Questions and Relative Clauses in Hindi. Dordrecht: Kluwer.

Dayal, Veneeta. 2002. Single-pair versus multiple-pair answers: Wh-in-situ and scope. Linguistic Inquiry 33(3). 512-520.

Dayal, Veneeta. 2018. Questions. Oxford: Oxford University Press.

Engdahl, Elisabet. 1980. The syntax and semantics of questions in Swedish: University of Massachusetts $\mathrm{PhD}$ dissertation.

Engdahl, Elizabet. 1986. Constituent questions. Dordrecht: Reidel.

Fox, Danny. 2012a. Multiple WH-questions: Uniqueness, pair-list and second order questions. Class notes for MIT seminars.

Fox, Danny. 2012b. Pair-list with universal quantifiers. MIT class notes.

Groenendijk, Jeroen \& Martin Stokhof. 1984. On the semantics of questions and the pragmatics of answers. Varieties of formal semantics 3. 143-170.

Hackl, Martin. 2000. Comparative quantifiers: Massachusetts Institute of Technology $\mathrm{PhD}$ dissertation.

Hagstrom, Paul Alan. 1998. Decomposing questions: Massachusetts Institute of Technology $\mathrm{PhD}$ dissertation.

Jacobson, Pauline. 1994. Binding connectivity in copular sentences. In Proceedings of Semantics and Linguistic Theory, vol. 4, 161-178.

Jacobson, Pauline. 1995. On the quantificational force of English free relatives. In Quantification in natural languages, 451-486. Springer.

Jacobson, Pauline. 1999. Towards a Variable-Free Semantics. Linguistics and Philosophy 22(2). 117-185.

Kotek, Hadas. 2014. Composing questions: Massachusetts Institute of Technology $\mathrm{PhD}$ dissertation.

Krifka, Manfred. 1991. Definite noun-phrases are not quantifiers. Linguistic Inquiry 
Getting quantifying-into questions uniformly

23(2). 156-163.

Lahiri, Utpal. 2002. Questions and answers in embedded contexts. Oxford University Press.

Link, Godehard. 1987. Generalized quantifiers and plurals. In Generalized Quantifiers, 151-180. Springer.

Moltmann, Friederike \& Anna Szabolcsi. 1994. Scope interactions with pair-list quantifiers. In Proceedings of North East Linguistics Society, vol. 24, 381-395.

Nicolae, Andreea Cristina. 2013. Any questions? polarity as a window into the structure of questions: Harvard University PhD dissertation.

Pafel, Jürgen. 1999. Interrogative quantifiers within scope. Linguistics and Philosophy 22(3). 255-310.

Partee, Barbara H. 1986. Noun phrase interpretation and type-shifting principles. In J. Groenendijk, D. de Jongh \& M. Stokhof (eds.), Studies in Discourse Representation Theory and the Theory of Generalized Quantifiers, 357-381. Foris, Dordrecht.

Preuss, Susanne Marie-Luise. 2001. Issues in the semantics of questions with quantifiers: Rutgers $\mathrm{PhD}$ dissertation.

Sharvit, Yael \& Jungmin Kang. 2017. Fragment functional answers. In Proceedings of Semantics and Linguistic Theory, vol. 26, 1099-1118.

Srivastav, Veneeta. 1991. Wh dependencies in Hindi and the theory of grammar: Cornell University Ithaca, New York PhD dissertation.

Szabolcsi, Anna. 1997. Quantifiers in pair-list readings. In Anna Szabolcsi (ed.), Ways of scope taking, Kluwer, Dordrecht.

Winter, Yoad. 2001. Flexibility Principles in Boolean Semantics. Cambridge, MA: MIT Press.

Xiang, Yimei. 2016. Interpreting questions with non-exhaustive answers: Harvard University Cambridge, Massachusetts PhD dissertation.

Xiang, Yimei. 2019. A hybrid categorial approach to question composition. Ms, Rutgers University.

Yimei Xiang

18 Seminary Place, Room 205

Department of Linguistics

Rutgers University

New Brunswick, NJ 08901

yimei.xiang@rutgers.edu 